\title{
Comparison of joint angles and electromyographic activity of the lower extremities during standing with wearing standard and revised high-heeled shoes: A pilot study
}

\author{
Young-Hyeon Bae ${ }^{\mathrm{a}, \mathrm{b}}$, Mansoo $\mathrm{Ko}^{\mathrm{b}}$ and Suk Min Lee ${ }^{\mathrm{c}, *}$ \\ ${ }^{a}$ Department of Physical Medicine and Rehabilitation, Samsung Medical Center, Seoul, Korea \\ ${ }^{\mathrm{b}}$ Department of Physical Therapy (DPT program), Angelo State University, San Angelo, TX, USA \\ ${ }^{\mathrm{c}}$ Department of Physical Therapy, Sahmyook University, Seoul, Korea
}

\begin{abstract}
Revised high-heeled shoes (HHSs) were designed to improve the shortcomings of standard HHSs. This study was conducted to compare revised and standard HHSs with regard to joint angles and electromyographic (EMG) activity of the lower extremities during standing. The participants were five healthy young women. Data regarding joint angles and EMG activity of the lower extremities were obtained under three conditions: barefoot, when wearing revised HHSs, and when wearing standard HHSs. Lower extremity joint angles in the three dimensional plane were confirmed using a VICON motion capture system. EMG activity of the lower extremities was measured using active bipolar surface EMG. Kruskal-Wallis one-way analysis of variance by rank applied to analyze differences during three standing conditions. Compared with the barefoot condition, the standard HHSs condition was more different than the revised HHSs condition with regard to lower extremity joint angles during standing. EMG activity of the lower extremities was different for the revised HHSs condition, but the differences among the three conditions were not significant. Wearing revised HHSs may positively impact joint angles and EMG activity of the lower extremities by improving body alignment while standing.
\end{abstract}

Keywords: Revised high-heeled shoes, joint angles, electromyographic activity, standing

\section{Introduction}

The habitual use of HHSs in daily routine may cause abnormal alignment of standing posture associated with the lower extremities and trunk deformities [1]. For example, increased lumbar lordosis, pelvic anteversion, and genu valgum are frequently observed among women who wear HHSs. Forward shifting of the center of gravity drives immediate changes in body alignment in order to control static balance during standing [1,2]. Such forward displacement of the center of gravity is associated with elevation of

\footnotetext{
${ }^{*}$ Corresponding author: Suk Min Lee, Department of Physical Therapy, Sahmyook University, Seoul, Korea. E-mail: leesm@ syu.ac.kr.
}

0928-7329/16/\$35.00 @ 2016 - IOS Press and the authors. All rights reserved

This article is published online with Open Access and distributed under the terms of the Creative Commons Attribution NonCommercial License. 

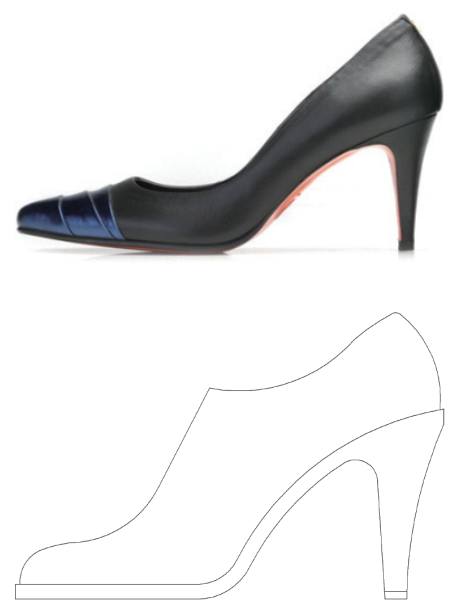

(a)
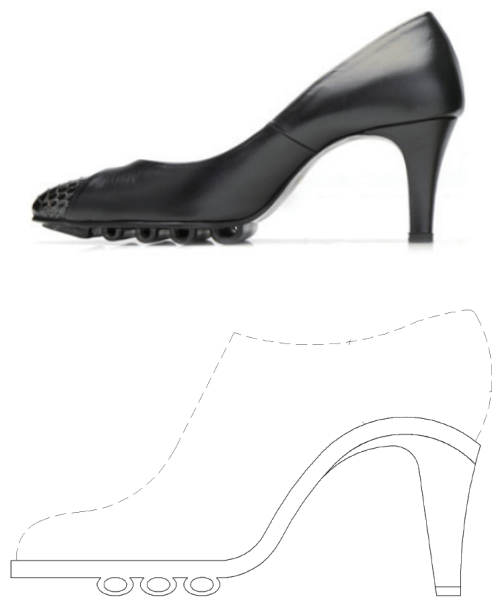

(b)

Fig. 1. High heeled shoes (HHSs) design (a) standard HHSs, and (b) revised HHSs.

the heel bone when wearing HHSs during upright standing [1]. Consequential overload on the forefoot frequently contributes to forefoot deformities, which may lead to postural instability [2].

Depending on the frequency of wearing standard HHSs, the location of the center of gravity may be shifted forward, resulting in temporary or permanent changes in body posture [3]. In order to stabilize body posture while standing, compensatory changes to drive effective motor response include increasing anterior pelvic tilt and/or changing the joint angles of the lower extremities [4]. Although excessive load on the forefoot and joint positioning of the lower extremities could return to normal without wearing HHSs, habitual wearing of HHSs may fixate abnormal postural alignment with shortened calf muscles and a tight Achilles tendon [3]. In addition, tibialis anterior (TA), quadriceps, and gastrocnemius muscles demonstrate increased electromyographic (EMG) activity with raised heel height, there were potentially contributed to instability of ankle joint [5-7].

Revised HHSs were designed for improve the faults of standard HHSs. There were try to drive a more effectively normal standing posture than standard HHSs through provides shock absorption of fore foot and backward decline of the wedge angle. Preliminary data have demonstrated that revised HHSs produce a $10 \%$ increase in body weight on the rear foot and a $10 \%$ decrease in body weight on the forefoot while standing [5]. In addition, revised HHSs are preferable to standard HHSs because there are result of normal improvement of foot pressure and a positive point of static balance [5]. Therefore, it would seem logical that the backward weight shift associated with revised HHSs may influence the joint angles and EMG activity of the lower extremities to maintain body posture. Accordingly, the investigated of compare revised and standard HHSs with regard to joint angles and EMG activity of the lower extremities during standing in adult women.

\section{Methods}

\subsection{Subjects and procedure}

Five healthy young women (mean: $21.3 \pm 1.5$ years, $164.3 \pm 2.4 \mathrm{~cm}, 54.8 \pm 2.1 \mathrm{~kg}$ ) were examined under 3 conditions in a randomized order: (1) barefoot, (2) while wearing $7 \mathrm{~cm}$ standard HHSs with, and 
(3) while wearing $7 \mathrm{~cm}$ revised HHSs (Fig. 1). All data were compared across the 3 study conditions, with a 1-day interval between conditions. Exclusion criteria were presence or history of neurologic or musculoskeletal disease. Women who were pregnant or had any psychological problem were also excluded. All subjects provided informed consent as required through the Institutional Review Board at Angelo State University.

\subsection{Measurement}

All measurements were obtained applying a VICON motion capture system (VICON, Oxford, UK). All standard HHSs were made by the same manufacturer to ensure the same foot pressure points. The revised HHSs were specifically designed implementing excellent shock absorption and backward decline of the wedge angle. Subjects became familiar with the test shoes. Subjects were permitted to wear shoes and walk around prior to conducting the standing balance test. Participants were requested to wear comfortable HHSs in the right size, which did not interrupt their natural walking pattern. Subjects stood in a natural posture, with their feet located with respect to sustain a $30^{\circ}$ angle between the right and left first toes with their arms relaxed alongside their body [8,9]. Participants remained standing for ten seconds without support, then rested for five seconds. This trial was repeated three times [3].

The following lower extremity joint angles were measured at a standing posture: joint angles of hip, knee, pelvic, and ankle at the sagittal (x-axis), frontal (z-axis), and transverse (y-axis) planes.

EMG activity of the lower extremities was evaluated using active bipolar surface EMG (Biometrics, Ladysmith, VA). Electrodes were placed over the peroneus longus (PL), tibalis anterior (TA), rectus femoris (RF), vastus lateralis (VLO), vastus medialis (VMO), medial gastrocnemius (MGCM), lateral gastrocnemius (LGCM), and soleus (SOL) muscles. EMG data were sampled at $1 \mathrm{kHz}$, then rectified and filtered for all muscles recorded. EMG activity of the TA, PL, VMO, VLO, RF, MGCM, LGCM, and SOL muscles during standing were analyzed separately. The root mean square values were calculated for all three standing conditions $[3,6,7]$.

\subsection{Analysis}

With respect to joint angles and EMG activity of the lower extremities, Kruskal-Wallis 1-way analysis of variance by rank was conducted to analyze differences among the three standing conditions. Significance of statistical was put up at $p<0.05$. All calculations were conducted applying SPSS version 21 (IBM Corporation, Armonk, NY, USA).

\section{Results}

Significant differences in joint angles were detected across all three standing conditions (Table 1). First, with respect to joint angles in the sagittal plane, both HHSs conditions had significantly greater plantar flexion and anterior pelvic tilt compared with the barefoot condition $(p<0.05)$. But, no significant differences were detected between both HHSs conditions. In the transverse plane, compete with the barefoot condition, the standard HHSs condition had significantly greater adduction $(p<0.05)$. However, there were no significant differences between both HHSs conditions. Lastly, in the frontal plane, both the standard and revised HHSs conditions had significantly greater internal rotation compared with the barefoot condition $(p<0.05)$. But, no significant differences were detected between both HHSs conditions. 
Table 1

Comparison between the three conditions (barefoot, standard HHSs, revised HHSs)

\begin{tabular}{|c|c|c|c|c|}
\hline \multicolumn{2}{|c|}{ Variable } & Bare foot & Standard HHSs & Revised HHSs \\
\hline Sagittal plane & $\begin{array}{l}\text { Ankle joint (angle) } \\
\text { Hip joint (angle) } \\
\text { Pelvic joint (angle) } \\
\text { Knee joint (angle) }\end{array}$ & $\begin{array}{r}-172.42 \pm 0.21 \\
-10.28 \pm 1.65 \\
-6.38 \pm 0.95 \\
14.75 \pm 0.31\end{array}$ & $\begin{aligned}-134.35 & \pm 0.20^{*} \\
-8.45 & \pm 0.10^{*} \\
-4.40 & \pm 0.58^{*} \\
15.93 & \pm 0.15^{*}\end{aligned}$ & $\begin{aligned}-147.49 & \pm 0.31^{* *} \\
-8.88 & \pm 0.59^{* *} \\
-5.44 & \pm 0.25^{* *} \\
15.39 & \pm 0.35^{* *}\end{aligned}$ \\
\hline Transverse plane & $\begin{array}{l}\text { Ankle joint (angle) } \\
\text { Hip joint (angle) } \\
\text { Pelvic joint (angle) } \\
\text { Knee joint (angle) }\end{array}$ & $\begin{aligned}-1.02 & \pm 0.15 \\
-15.37 & \pm 0.20 \\
-3.69 & \pm 0.15 \\
6.78 & \pm 0.20\end{aligned}$ & $\begin{aligned} 1.41 & \pm 0.10^{*} \\
-12.61 & \pm 0.15^{*} \\
-1.75 & \pm 0.20^{*} \\
3.42 & \pm 0.10^{*}\end{aligned}$ & $\begin{aligned} 1.09 & \pm 0.58 \\
-15.80 & \pm 0.57 \\
-2.45 & \pm 0.56 \\
6.31 & \pm 0.30\end{aligned}$ \\
\hline Frontal plane & $\begin{array}{l}\text { Ankle joint (angle) } \\
\text { Hip joint (angle) } \\
\text { Pelvic joint (angle) } \\
\text { Knee joint (angle) }\end{array}$ & $\begin{aligned} &-7.01 \pm 0.93 \\
&-62.59 \pm 0.29 \\
& 1.49 \pm 0.66 \\
& 59.67 \pm 1.97 \\
&\end{aligned}$ & $\begin{aligned} 6.33 & \pm 0.26^{*} \\
-61.25 & \pm 0.25^{*} \\
4.79 & \pm 0.47^{*} \\
66.22 & \pm 0.20^{*}\end{aligned}$ & $\begin{aligned} 5.92 & \pm 0.58^{* *} \\
-62.17 & \pm 0.20^{* *} \\
2.53 & \pm 1.58^{* *} \\
62.12 & \pm 0.35^{* *}\end{aligned}$ \\
\hline
\end{tabular}

HHSs: High heeled shoes, ${ }^{*}$ Significant difference between the standard HHS and bare foot $(P<0.05),{ }^{* *}$ Significant difference between the revised HHS and bare foot $(P<0.05)$.

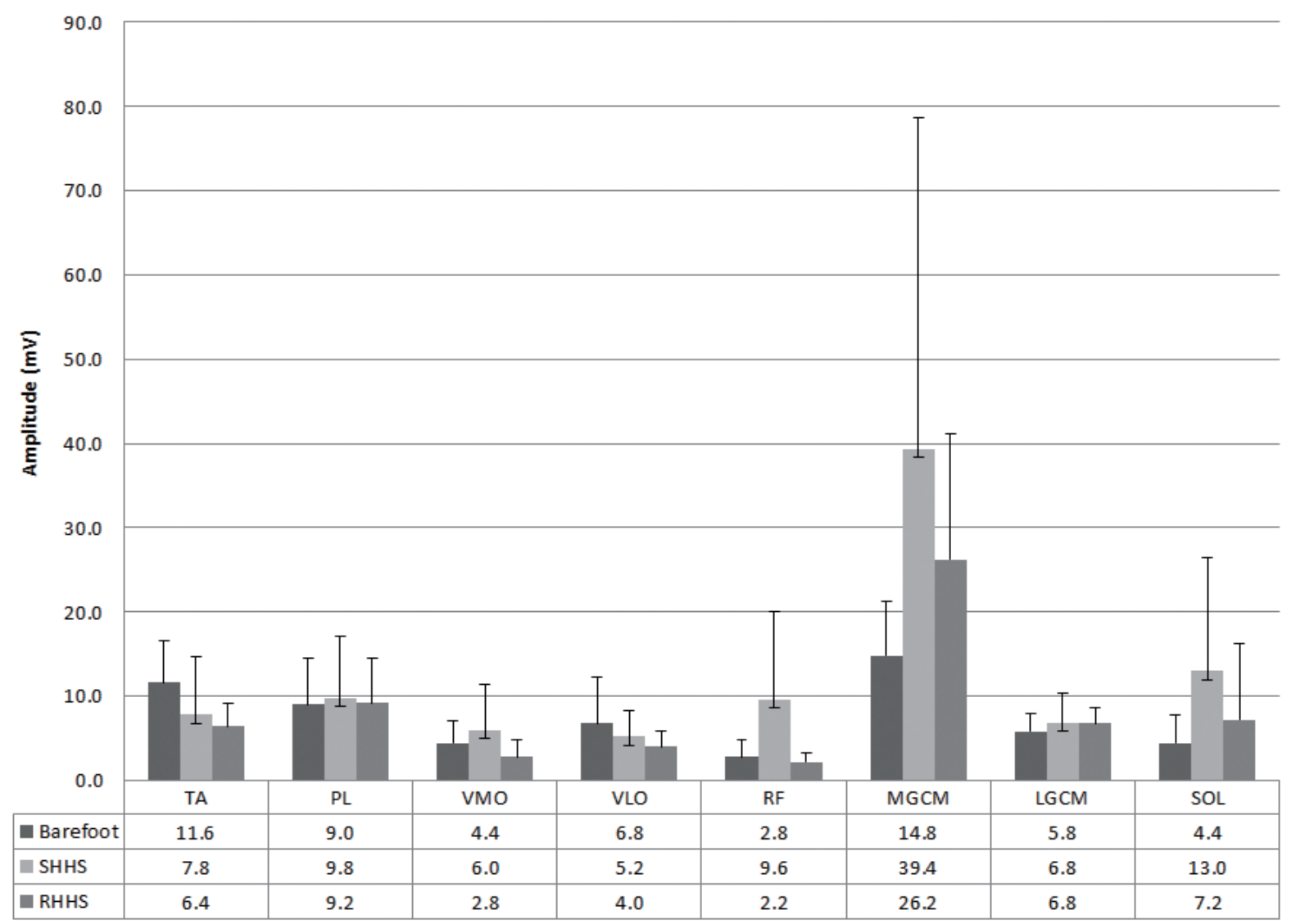

Fig. 2. Comparison of EMG activity between the 3 conditions. High-heeled shoes (HHS), Tibialis anterior(TA), Peroneous loguns (PL), Vastus lateralis (VLO), Vastus medialis (VMO), Rectus femoris (RF), Medial gatrocnemius (MGCM), Lateral gastrocnemius (LGCM), Soleus (SOL) muscles. 
The normalized EMG activity data collected during standing are summarized in Fig. 2. The PL, VMO, RF, MGCM, LGCM and SOL EMG activities were highest for the SHHS condition than for other conditions. And the TA and VLO were lowest for the RHHS condition than for other than conditions. But all EMG activities were not significant difference between the 3 conditions.

\section{Discussion}

This study investigated the effect of revised HHSs, which implement tunnel shock absorption to achieve better body alignment and enhance body posture. It should be noted that most of the participants commented that the revised HHSs successfully supported the arch of the foot, compared with the standard HHSs, while standing. Preliminary data of 15 healthy adult women have shown that revised HHSs produce a $10 \%$ weight shift to the rear foot compared with standard HHSs [5]. In addition, revised HHSs are preferable to standard HHSs because they result of normal improvement of foot pressure and a positive point of static balance [5]. Because revised HHSs provide rearward decline of the wedge angle and thicker forefoot cushioning, we believe that the joint angles of the lower extremities can be repositioned in response to significant weight distribution on the rear foot while standing.

Standing with wearing standard HHSs causes weight shift of forward, with a significant increase in plantar pressure on the forefoot than on the rear foot [1]. In general, habitual wearing of standard HHSs got to produce ankle plantar flexion, while the knee has been shown to be more flexed with increased heel height $[7,10,11]$. Activity of the TA, quadriceps, and gastrocnemius muscles also increases with raised heel height, there were potentially contributed to instability of ankle joint $[5-7,10]$.

A previous study indicated that revised HHSs cause significantly increasing of back foot pressure and decreasing of forefoot pressure than standard HHSs. And, revised HHSs got to more effectively normal standing posture and positive point of static balance by provides shock absorption of fore foot and backward decline of the wedge angle [5].

Although there were no significant differences between the revised and standard HHSs conditions in this pilot study, the lower extremity joint angles while wearing the revised HHSs were closer to normal. Revised HHSs tend to normalize static balance during standing by preventing motor actions such as increased or decreased flexion, adduction, or internal rotation of the pelvis, knee, hip, or ankle joints [1,2]. Therefore, wearing revised HHSs may positively influence stability of the joint angles and EMG activity of the lower extremities by improving body alignment while standing.

Because this pilot study used a small sample size and a cross-sectional design, one outlier may narrow or broaden the effect size, which could negatively influence any statistical significance (type II error). This study have a little limitation which the effects observed for the improvement of revised HHSs condition were not according on a measurement methods of sufficient.

\section{Acknowledgement}

This study was supported by Sahmyook University.

\section{References}

[1] A.M. Silva AM, G.R. de Siqueira and G.A. da Silva, Implications of high-heeled shoes on body posture of adolescents, Rev Paul Pediatr 31 (2013), 265-271. 
[2] D.C. Kerrigan, M.K. Todd and P.O. Riley, Knee osteoarthritis and high-heeled shoes, Lancet 351 (1998), 1399-1401.

[3] K.A. Opila, S.S. Wagner, S. Schiowitz, et al., Postural alignment in barefoot and high-heeled stance, Spine 13 (1988), 542-547.

[4] J. Yu, J.T. Cheung and D.W. Wong, et al., Biomechanical simulation of high-heeled shoe donning and walking, J Biomech 46 (2013), 2067-2074.

[5] Y.H. Bae, M.S. Ko, Y.S. Park, S.M. Lee, Effect of revised high-heeled shoes on foot pressure and static balance during standing, J Phys Ther Sci 27 (2015), 1129-1131.

[6] A. Mika, L. Oleksy, P. Mika, et al., The influence of heel height on lower extremity kinematics and leg muscle activity during gait in young and middle-aged women, Gait Posture 25 (2012), 677-680.

[7] A. Mika, L. Oleksy, E. Mikolajczyk, et al., Changes of bioelectrical activity in cervical paraspinal muscle during gait in low and high heel shoes, Acta Bioeng Biomech 13 (2011), 27-33.

[8] T.S. Kapteyn, W. Bles, C.J. Njiokiktjien, et al., Standardization in platform stabilometry being a part of posturography, Agressologie 24 (1983), 321-326.

[9] T.E. Prieto, J.B. Myklebust, R.G. Hoffmann, et al., Measures of postural steadiness: Differences between healthy young and elderly adults, IEEE Trans Biomed Eng 43 (1996), 956-966.

[10] M.G. Blanchette, J.R. Brault, C.M. Powers, The influence of heel height on utilized coefficient of friction during walking, Gait Posture 34 (2011), 107-110.

[11] C.J. Ebbeling, J. Hamill, J.A. Crussemeyer, Lower extremity mechanics and energy cost of walking in high-heeled shoes, J Orthop Sports Phys Ther 19 (1994), 190-196. 